\title{
Positive selection in development and growth rate regulation genes involved in species divergence of the genus Radix
}

\author{
Barbara Feldmeyer ${ }^{1,2^{*}}$, Bastian Greshake ${ }^{1,3}$, Elisabeth Funke ${ }^{1}$, Ingo Ebersberger ${ }^{3}$ and Markus Pfenninger ${ }^{1}$
}

\begin{abstract}
Background: Life history traits like developmental time, age and size at maturity are directly related to fitness in all organisms and play a major role in adaptive evolution and speciation processes. Comparative genomic or transcriptomic approaches to identify positively selected genes involved in species divergence can help to generate hypotheses on the driving forces behind speciation. Here we use a bottom-up approach to investigate this hypothesis by comparative analysis of orthologous transcripts of four closely related European Radix species.

Results: Snails of the genus Radix occupy species specific distribution ranges with distinct climatic niches, indicating a potential for natural selection driven speciation based on ecological niche differentiation. We then inferred phylogenetic relationships among the four Radix species based on whole mt-genomes plus 23 nuclear loci. Three different tests to infer selection and changes in amino acid properties yielded a total of 134 genes with signatures of positive selection. The majority of these genes belonged to the functional gene ontology categories "reproduction" and "genitalia" with an overrepresentation of the functions "development" and "growth rate".

Conclusions: We show here that Radix species divergence may be primarily enforced by selection on life history traits such as (larval-) development and growth rate. We thus hypothesise that life history differences may confer advantages under the according climate regimes, e.g., species occupying warmer and dryer habitats might have a fitness advantage with fast developing susceptible life stages, which are more tolerant to habitat desiccation.
\end{abstract}

Keywords: Adaptive sequence evolution, Positive selection, Transcriptomics, RNA-Seq, Mollusks, Adaptation, Reproductive isolation

\section{Background}

Understanding the driving forces of speciation processes is a primary goal of evolutionary biology [1]. Various mechanisms can lead to the evolution of reproductive isolation between populations and ultimately to speciation $[2,3]$. One of these mechanisms is ecology-driven diversifying selection imposed by the demands of adapting to niche-specific biotic and abiotic factors [3-5]. One major target of these adaptive processes is the evolution of life history traits such as lifespan, growth, development and

\footnotetext{
* Correspondence: barbara.feldmeyer@senckenberg.de

${ }^{1}$ Molecular Ecology Group, Senckenberg Biodiversity and Climate Research Centre (BiK-F), Georg-Voigt-Str. 14-16, Frankfurt am Main 60325, Germany Evolutionary Biology, Johannes Gutenberg University Mainz, Müllerweg 6, Mainz 55099, Germany

Full list of author information is available at the end of the article
}

plasticity $[6,7]$. According to life history theory organisms try to optimize their survival and reproduction, thus their fitness, by following a species specific pattern in allocating resources to life history traits $[6,7]$. For example developmental rates and growth are dependent on prevailing ecological conditions, and influence size and age at maturity $[6,7]$. Ecological conditions affecting developmental timing and growth include predator prevalence $[8,9]$, nutrition $[8,10]$, as well as climate variables $[11,12]$.

Ultimately, most local environmental variables are affected by climate, which is especially the case in fresh water systems where climate is a major driver of abiotic and biotic processes [13]. Apart from the obvious impact of precipitation on freshwater systems, the temperature of a given stretch of stream or river varies seasonally, weekly and daily and is dependent on a number of factors, including air temperature, but also depth, flow,

\section{Ciomed Central}

(c) 2015 Feldmeyer et al. Open Access This article is distributed under the terms of the Creative Commons Attribution 4.0 International License (http://creativecommons.org/licenses/by/4.0), which permits unrestricted use, distribution, and reproduction in any medium, provided you give appropriate credit to the original author(s) and the source, provide a link to the Creative Commons license, and indicate if changes were made. The Creative Commons Public Domain Dedication waiver (http://creativecommons.org/publicdomain/zero/1.0/) applies to the data made available in this article, unless otherwise stated 
elevation, latitude, water input (overland runoff versus groundwater) and the type and extent of riparian vegetation $[14,15]$. Variation in air temperature not only leads to changes in water temperature but might also lead to alterations in additional water body conditions such as reduced oxygen availability, increased salinity and the desiccation of the water body.

Due to the large number of ecological variables organisms are exposed to, it is not always possible to accurately determine the respective selective regimes acting in a system. It is thus often difficult and sometimes misleading to reconstruct post hoc the evolutionary forces that lead to the separation even of closely related lineages. "Reverse ecology" is one way to obtain plausible hypotheses on the driving processes of reproductive isolation [16]. This approach uses comparative genomic data to identify genes and their functions whose evolution has been driven by positive selection $[16,17]$. Under the assumption that those genes most likely code for important ecological phenotypes [18] they can help to infer the response of organisms to their environment. Recent developments in next-generation sequencing technologies have led to the availability of genomic resources for many nonmodel organisms, allowing us to apply the reverse ecology approach to a wider range of organisms [16].

In this study we make use of the reverse ecology approach to gain insights into the role of ecological factors leading to species divergence in the pond snail genus Radix Montfort 1810. The genus Radix is part of the Lymnaeidae family (Basommatophora) and contains airbreathing, simultaneously hermaphroditic snail species, which are distributed throughout the Paleartic [19, 20]. Shell size, coloration and genital anatomy are highly variable even within species [21, 22] and often overlap between species, rendering morphological delimitation of species rather difficult [21, 23, 24]. A COI based barcoding effort based on specimen from North Western Europe resulted in five "Molecularly defined Taxonomic Units" (MOTU1-5), some of which could be attributed to existing species [23]. The type of water bodies occupied by the different species varies from deep, permanent waters (R. auricularia), to clear and cold streams (MOTU5), to slow flowing rivers, ditches and ponds ( $R$. balthica and MOTU3) $[19,20,23]$. Moreover, the distinct distribution ranges [25] may indicate climatic niche differences among species, mirrored on the genomic level. We may thus expect to find positively selected genes with biological functions related to energy metabolism, desiccation resistance or salinity tolerance. Indeed, climate is known to affect such ecologically relevant fitness traits in other aquatic gastropods [26-28].

We use an RNA-Seq approach to obtain the transcriptomes of $R$. auricularia, MOTU3 and MOTU5 to obtain ortholog gene sequences for the selection analyses. In addition, we reconstruct the full mitochondrial genomes for each of the four species, since mitochondrial genes are known to play a central role in temperature dependent metabolism of ectotherms [29]. Three different tests for selection are applied to identify possible candidate genes involved in species divergence in the genus Radix.

\section{Results}

For this study we sequenced the transcriptomes for three Central European Radix species. MOTU3 and R. auricularia were sequenced on the Roche 454 and MOTU5 on the Illumina HiSeq 2000 platform. The sequencing effort resulted in more than 400,000 to over 100 Mio reads respectively. Accession numbers, summary on the number of reads, as well as summary statistics on the de novo assembled contigs can be found in Additional file 1. The transcriptome and assembly information of $R$. balthica has been published in a previous study [30].

\section{Mitochondrial genomes}

As intergenic regions in mitochondrial genomes are rather small, it was possible to assemble a large part of the mt-genomes based on transcriptome contigs. The remaining gaps were closed by additional Sanger sequences, which we also used for sequence verification. The length of the mt-genomes was $13,745 \mathrm{bp}$ in $R$. auricularia [GenBank:KP098540]; 13,832 bp in MOTU5 [GenBank:KP098539]; 13,963 bp in MOTU3 [GenBank:KP098538] and 13,983 bp in $R$. balthica [GenBank:KP098541]. Mitochondrial genome nucleotide divergence ranges from $0.04 \%$ between the two sister species $R$. balthica and MOTU3 and $0.24 \%$ between MOTU3 and $R$. auricularia. The location and orientation of all 13 protein coding genes and the two rRNAs is identical in all four species. In contrast, the gene order within a cluster of seven tRNAs between the COII and ATP8 is variable (Fig. 1). Both observations resemble the findings of a previous comparative analysis of mt-genomes in a study of three basommatophoran genera [31]. Using $R$. auricularia as reference, the tRNA-Cys was shifted two positions towards the 5 '-end of the tRNA cluster in MOTU5. Again with $R$. auricularia as reference, tRNA-Trp shifted two positions towards the $5^{\prime}$-end in both, $R$. balthica and MOTU3 (Fig. 1).

\section{Phylogenetic relationships}

With Biomphalaria glabrata as an outgroup, both phylogenetic reconstructions lead to the same phylogenetic relationship among species (Bayesian reconstruction based on mt-genes only (Additional file $2 \mathrm{~A}$ ); reconstruction with additional 23 nuclear loci (Additional file 2 B)). The trees consistently identify $R$. balthica and 


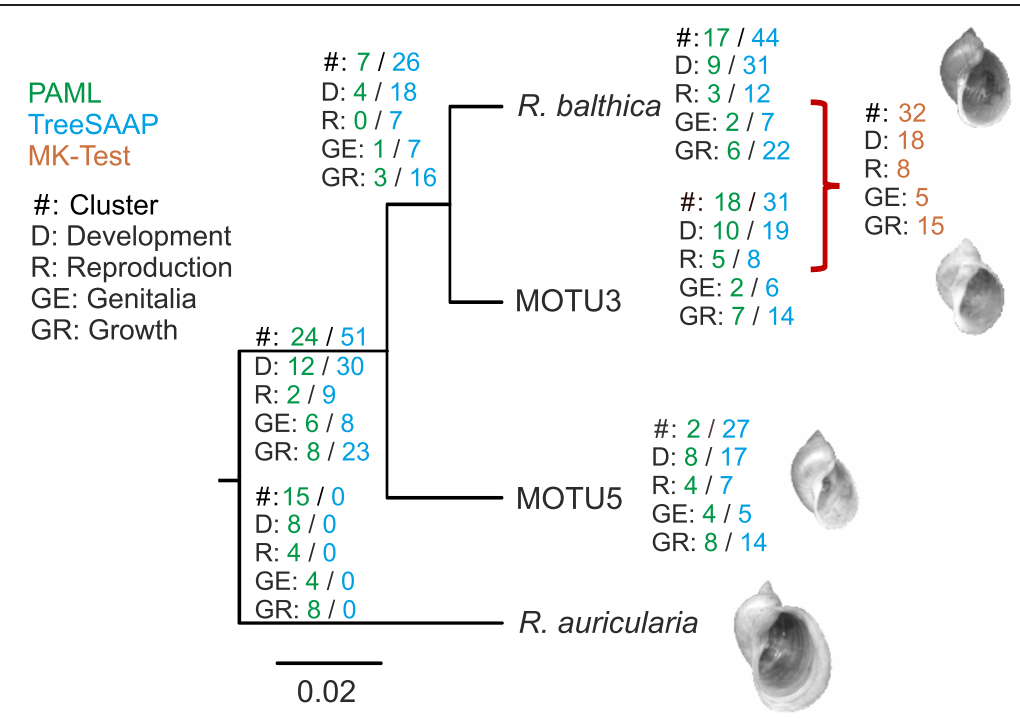

Fig. 1 Schematic overview of the gene order in mitochondrial genomes of the four Radix species investigated. Gene orientation is indicated by arrows. Inferred tRNA rearrangements are indicated by arrows in the detailed section. Tree topology inferred from phylogenetic analysis (Additional file 2)

MOTU3 as sister species, as expected from Pfenninger et al. [23]. R. auricularia constitutes the earliest branching lineage among the four Radix species.

\section{Climatic niche differentiation}

An analysis of 32 climatic variables [32] at 228 sampling sites of the four Radix species was conducted to determine climate niche differences between species. A principle component analysis revealed significant differences in PCA1 (temperature: $\mathrm{F}_{(3,225)}=4.5 ; p=0.005$ ) as well as PCA2 (precipitation: $\mathrm{F}_{(3,225)}=6.66 ; p=0.0015$ ) between the distribution areas of the four Radix species. MOTU3 inhabits the warmest and driest region, while MOTU5 can be found in the coldest habitat with the highest amount of precipitation (Additional file 3).

\section{Ortholog gene clusters and tests for positive selection}

We used the tool HaMStR [http://www.sourceforge.net/ projects/hamstr] [33] to search for ortholog sequences in the Radix transcriptome data. For 354 genes an ortholog was detected in all four species. For an additional 236 genes we detected orthologs in each of the two sister species $R$. balthica \& MOTU3.

Three different methods (PAML, McDonald-Kreitman Test, TreeSAAP) were applied to test for positively selected genes among the four species. In total 134 clusters were identified as positively selected by at least one of the three methods (Additional file 4; Fig. 2). Of these, 116 were identified by a single method only, and five clusters by all three methods (Table 1). With the PAML method 56 clusters (out of 354 ortholog clusters) were classified as positively selected $(\omega>1)$. The number of selected clusters per branch ranged from two in MOTU5 to 18 in MOTU3 (Fig. 1). There was no difference in the frequency of the four most abundant functional gene ontology categories among the branches (larval development: $X^{2}=1.91, \mathrm{df}=5, p=0.86$; (regulation of) growth (rate): $X^{2}=1.83, \quad \mathrm{df}=5, \quad p=0.87$; reproduction: $X^{2}=6.35, \mathrm{df}=5, p=0.27$; hermaphrodite genitalia development: $X^{2}=3.08, \mathrm{df}=5, p=0.69$ ), indicating their relevance in species divergence among all four species. With TreeSAAP 69 unique clusters fulfilled the criteria for positive selection. The number of positively selected TreeSAAP clusters per branch ranged from zero on the branch leading to $R$. auricularia to 44 on the branch to $R$. balthica (Fig. 1). The pair-wise comparison between the two sister species $R$. balthica and MOTU3 was based on 590 ortholog clusters, of which 32 were identified as positively selected according to the McDonald-Kreitman Test $(D n / D s>P n / P s)$. None of the 134 genes with signatures of selection are of mitochondrial origin.

\section{Functional enrichment analyses}

We conducted an enrichment analysis to test for overrepresentation of functional categories among the complete set of positively selected genes and among subsets according to branches. The construction of clusters with HaMStR is based on a set of core-orthologs which are evolutionary conserved genes. The sequences/contigs used in the analyses are transcriptome based and hence a subset of all genes. We might thus introduce a 


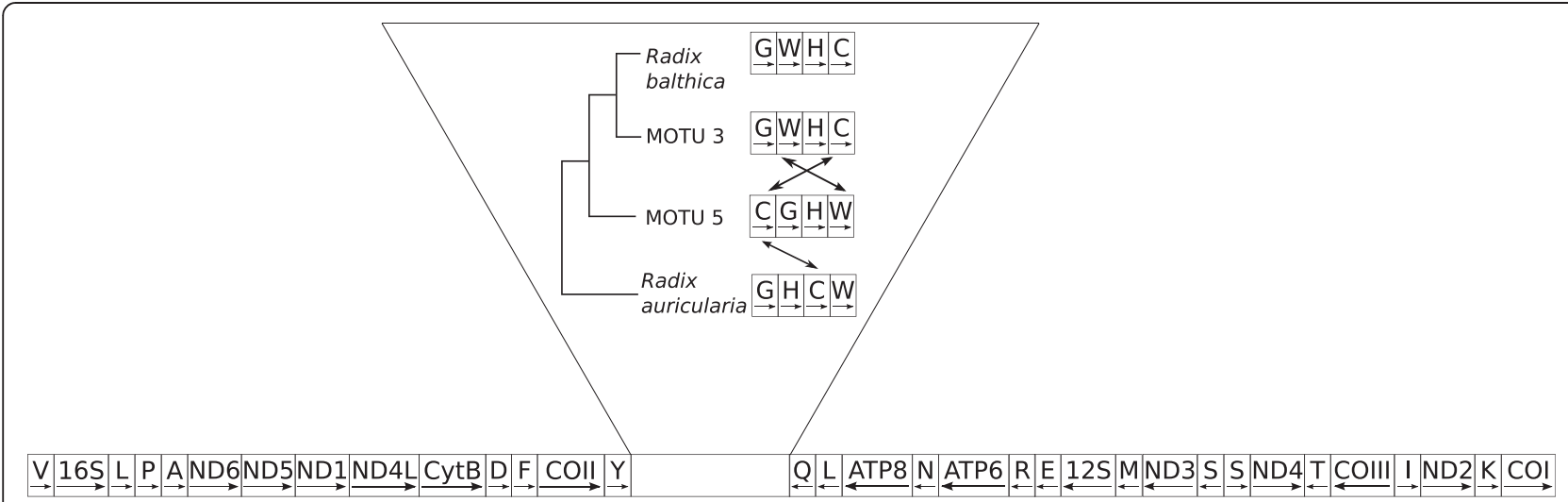

Fig. 2 Number of selected gene clusters (\#) with respective association to the four most abundant functional categories. Selected genes were identified with three different methods: PAML (green); TreeSAAP (blue); MK-Test (brown); D = "development" (GO:0009792/0002119/0009792); $\mathrm{R}=$ "reproduction" (GO:00000003/ 0018991); GE = "genitalia development" (GO:0048806/0040035); GR= "Growth" (GO:0040007/0040010); $\mathrm{n}=$ node\# as referred to in the selection analyses. Note that a single cluster can belong to multiple functional categories. (Topology inferred from phylogenetic analysis (Additional file 2))

systematic bias in functional categories associated to subsets of contigs. To account for these possible biases we conducted enrichment analyses with the set of contigs with and without signatures of selection against the background set of all 590 contigs each. Functional categories identified in both sets of contigs might be due to the above mentioned systematic bias and are excluded from further discussion. In the clusters without signature of selection, 14 functional categories are overrepresented $($ FDR $<0.05)$ when tested against the background set (Table 2; further details in Additional file 5). Only two categories overlap between the cluster sets with and without signatures of selection, namely "growth" and "post-embryonic development".

Excluding the two overlapping functional categories, seven categories are exclusively overrepresented in the complete selected cluster set (all four species; all three selection tests), which are mainly involved in the regulation of growth (-rate) and larval development (Table 2). When excluding the MK-test identified genes based on MOTU3 and $R$. balthica only, the development related functions dropped out, but the growth (-rate) related functional categories are still overrepresented in this smaller cluster-set (all four species; PAML + TreeSaap)

Table 1 Summary of clusters identified as positively selected by all three methods (MK-Test results not shown in table which

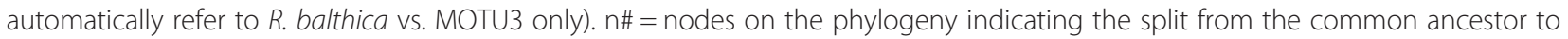
respective species (see Fig. 2 for details)

\begin{tabular}{|c|c|c|c|c|}
\hline Cluster & TreeSAAP & Paml & Annotation & GO terms \\
\hline 111248 & $\mathrm{n} 7->$ R. balthica & n7 - > Motu3 & $\begin{array}{l}\text { mannosyl-oligosaccharide alpha- -mannosidase } \\
\text { isoform }\end{array}$ & determination of adult lifespan \\
\hline 111317 & $\begin{array}{l}\mathrm{n} 7->R \text {. balthica } \\
\mathrm{n} 5->\mathrm{n} 6\end{array}$ & n7 - > Motu3 & signal recognition particle $54 \mathrm{kda}$ protein & nematode larval development reproduction \\
\hline 111407 & $\begin{array}{l}\mathrm{n} 7->\text { R. balthica } \\
\mathrm{n} 6->\text { Motu5 } \\
\mathrm{n} 5->\mathrm{n} 6\end{array}$ & $\begin{array}{l}\text { n7 - > Motu3 } \\
\text { n5 - >n6 }\end{array}$ & NA & NA \\
\hline 111416 & $\mathrm{n} 5->\mathrm{n} 6$ & $\begin{array}{l}\mathrm{n} 7->\text { R. balthica } \\
\mathrm{n} 7->\text { Motu3 } \\
\mathrm{n} 6->\mathrm{n} 7 \\
\mathrm{n} 5->\mathrm{n} 6\end{array}$ & $\begin{array}{l}\text { dolichyl-diphosphooligosaccharide-protein } \\
\text { glycosyltransferase } 48 \text { kda subunit }\end{array}$ & $\begin{array}{l}\text { positive regulation of growth rate } \\
\text { larval development }\end{array}$ \\
\hline 111457 & $\begin{array}{l}\mathrm{n} 7->\text { R. balthica } \\
\mathrm{n} 6->\text { Motu5 } \\
\mathrm{n} 6->\mathrm{n} 7 \\
\mathrm{n} 5->\mathrm{n} 6\end{array}$ & $\begin{array}{l}\text { n7 }->\text { Motu3 } \\
\text { n5 - > n6 }\end{array}$ & $\begin{array}{l}\text { eukaryotic translation initiation } \\
\text { factor } 3 \text { subunit e }\end{array}$ & $\begin{array}{l}\text { genitalia development embryo/larval } \\
\text { development }\end{array}$ \\
\hline
\end{tabular}


Table 2 Summary of enriched functional categories (FDR < 0.05) overrepresented in cluster-sets with and without selection. In italic, functional categories enriched in both types of clusters

\begin{tabular}{|c|c|c|}
\hline \multirow{2}{*}{$\begin{array}{l}\text { Without signature of selection } \\
\text { all three tests }\end{array}$} & \multicolumn{2}{|l|}{ With signature of selection } \\
\hline & all three tests & without MK-test \\
\hline translation & growth & regulation of growth \\
\hline cellular protein metabolic process & regulation of growth & growth \\
\hline growth & positive regulation of growth & positive regulation of biological process \\
\hline cellular process involved in reproduction & positive regulation of biological process & positive regulation of growth \\
\hline mitochondrial ATP synthesis coupled electron transport & positive regulation of growth rate & positive regulation of growth rate \\
\hline post-embryonic development & regulation of growth rate & regulation of growth rate \\
\hline meiotic cell cycle & nematode larval development & \\
\hline ribosome & larval development & \\
\hline ribonucleoprotein complex & post-embryonic development & \\
\hline \multicolumn{3}{|l|}{ cytoplasmic part } \\
\hline \multicolumn{3}{|l|}{ cytoplasm } \\
\hline \multicolumn{3}{|l|}{ macromolecular complex } \\
\hline \multicolumn{3}{|l|}{ structural constituent of ribosome } \\
\hline structural molecule activity & & \\
\hline
\end{tabular}

(Table 2). In the cluster set with the 32 selected genes identified between $R$. balthica and MOTU3 by the MKTest, none of the functions appears to be overrepresented.

With respect to our initial hypothesis that genes involved in metabolism could play a role in species divergence, we could not identify any metabolism related overrepresented functional category in the selected gene clusters. However, out of the 134 genes with signs of positive selection, seven are involved in metabolism related functions such as "oxidation reduction process" and "metabolic process". Three of these seven show signs of selection on the branch to R. balthica, and two more between $R$. balthica and MOTU3 by the MK-Test. Thus only two of the seven genes are found on branches other than $R$. balthica, namely $R$. auricularia and the branch leading from $R$. auricularia to the other species.

\section{Discussion}

Understanding the underlying forces leading to species divergence is of great interest in evolutionary biology. One possible mechanism is ecological speciation, defined as the evolution of reproductive isolation between populations as a result of ecologically-based divergent natural selection [5]. We set out to determine genes possibly involved in species divergence among four freshwater snail species of the genus Radix inhabiting distinct distribution ranges. We hypothesized that the distribution ranges differ in climatic niche properties, leading to species divergences based on selection on species specific physiological traits. We show here, that indeed distribution ranges of the four species differ significantly in their climatic conditions, mainly in precipitation and temperature. However, genes relevant in physiology and metabolic related processes only seem to play a minor role.

As mitochondrial genes are major players in the respiratory cascade and are thus involved in metabolic processes [34], selection on some of these genes involved in oxidative phosphorylation may lead to adaptation to different environments [29, 35, 36]. The comparison of the four mitochondrial genomes revealed rather large congruence among species. While the gene order of protein coding genes is identical among species, the position of four tRNAs (GWHC), within a cluster of seven tRNAs varies between species. Even though the Radix species investigated here inhabit distinct climatic niches, none of the mitochondrial genes, central in energy metabolism, shows signatures of selection among the Radix species.

\section{Function and relevance of nuclear genes with signs of positive selection}

In contrast to the mitochondrial genes, we were able to identify 134 nuclear genes with signs of positive selection between the four species. Seven of these genes are related to metabolism and oxidation processes and thus might indicate physiological adaptations in respect to temperature differences. Many of these were detected between the two sister species $R$. balthica and MOTU3. This corroborates findings of a recent study investigating a hybrid zone between these two species, where precipitation and temperature were the environmental factors explaining species and hybrid zone distribution [37]. Amongst the genes with signs of positive selection we 
also identified functions related to reproduction and hermaphrodite genitalia development. Genitalia morphology is one of the most species specific characteristics, and plays a major role in species divergence [38-40]. Genitalia morphology is known to be subjected to rapid evolution through sexual selection [41], which is especially wide spread in animals with internal fertilization [39]. Our findings might indicate a role of ongoing active enforcement of mechanical reproductive isolation in the Radix complex, albeit considerable inter- and intraspecific morphological variations for the bursa position and bursa duct $[21,42]$.

However, the most frequent and overrepresented functions among the selected genes are larval development and (regulation of-) growth (-rate). These functions are well known life-history traits. Based on life-history theory, it is generally assumed that organisms are facing numerous trade-offs due to a limited amount of resources $[6,7]$. For example, long lived organisms usually produce only a small number of offspring, whereas short lived organisms produce comparatively many. In general, body size is positively correlated with survival and fecundity, however larger size often requires additional time for growth, thus leading to older age at maturity $[6,7]$. On the other hand, when the risk of mortality increases in the larval environment, faster development at the cost of smaller body size may still be favored $[6,43]$. Thus depending on the available resources and environmental conditions, organisms need to trade off which of the trajectories they will follow.

With respect to larval development, two factors play a crucial role; timing and rate. Developmental timing (also called heterochrony) is a complex trait [44]. It can lead to shifts in timing of gene expression and result in morphological alterations [44, 45], even though the underlying genes are shared among distantly related groups [46]. In fact, recent publications on several gastropod species, and Radix in particular, have uncovered interand intraspecific variation in embryonic development $[47,48]$, and a correlation between developmental timing and genetic differentiation in $R$. balthica populations [48]. Thus the variation in developmental timing in combination with genetic differentiation could serve as raw material for natural selection and drive species divergence [48].

Developmental rate, the time elapsed from embryo to the reproductive phase, shows considerable variation in natural populations [49]. Developmental rate genes have pleiotropic effects on several adult traits and the action of most of them is sensitive to temperature during development [50]. In this respect the role of environmental conditions has been suggested as a selective force driving the differentiation of embryonic development in a number of species, such as nematodes [51, 52], lizards
[53] and various gastropod taxa [54]. Interestingly, developmental rates are under strong selection in a number of aquatic organisms facing the threat of desiccation, since it allows organisms and their live stages to avoid unfavorable conditions [55, 56]. For example Killifish embryos decrease their developmental rates leading to a developmental arrest in early stages of development in response to desiccation [56]. On the other hand, many amphibians accelerate development to avoid desiccation, often at the cost of body size [55 and authors therein]. In gastropod species life stage, as well as individual size have a large intra-specific effect on desiccation resistance [57-59]. In snails, an increase in shell size can be a mechanism of desiccation resistance due to reduced water loss as a result of a better surface-volume ratio $[58,59]$. From these examples it becomes clear that the interplay between developmental timing, the rate of development, growth rate and size may indeed play an important role in shaping the fitness landscape of aquatic organisms.

As mentioned before, the hybrid zone between the sister species $R$. balthica and MOTU3 is best explained by local climatic factors, namely precipitation and temperature [37]. The great number of positively selected genes with functions in development and growth (-rate) together with the above examples and the pronounced climatic differences between the distribution areas of the four Radix species, leads to the hypothesis that species divergence is mainly driven by a trade-off in life-history parameters leading to niche adaptation. For example in hot and dry environments a quick development with fast growth might prevent susceptible larvae and small individuals from desiccation. Cold environments, on the other hand, might lead to slow development, smaller shell and smaller clutch sizes, however might result in longer lifespan [60].

\section{Conclusions}

Based on pronounced differences in climatic niche properties between the four Radix species, we expected to find genes involved in physiological and metabolic traits to be under selection. However, the results from our study suggest that species divergence in Radix is mainly driven by selection on life-history traits, mainly development and growth, rather than physiological properties. As climate is known to have direct effects on various water body properties and therefore also on ecologically relevant fitness traits in aquatic gastropods, such as desiccation tolerance [26-28], we hypothesize that developmental rate and individual size might be of high adaptive importance in the genus Radix. The bottom up approach applied here provides a number of unexpected results and new hypotheses, which can now be tested in follow up functional experiments. This reverse ecology 
approach has thus proven valuable for hypotheses generation in evolutionary research.

\section{Methods}

\section{Sample collection and treatments}

Snails for transcriptome analyses were originally collected from different sites in France (MOTU3), Switzerland (MOTU5) and Germany ( $R$. auricularia) between 2005 and 2011 (Additional file 6) and kept in water basins untill the start of this project. Information on treatment and sequence generation for $R$. balthica snails is reported in a previous study and documented in Feldmeyer et al. [30]. We placed individual snails in different temperature treatments. In 2011 snails were transferred to $500 \mathrm{ml}$ glass jars and underwent one of six treatments, with two individuals per population, per treatment: Three days at $10{ }^{\circ} \mathrm{C}$ or $30^{\circ} \mathrm{C}$ with and without aeration, 10 min temperature shock at either $4{ }^{\circ} \mathrm{C}$ or $36^{\circ} \mathrm{C}$ before storage in RNAlater (Qiagen). RNA was isolated following the RNeasy maxi kit protocol (Qiagen). cDNA production, normalization, and sequencing was performed by GenXPro $\mathrm{GmbH}$ (Frankfurt, Germany). Due to technical issues and different time points of sequencing, the three libraries were sequenced on different platforms, MOTU3 $+R$. auricularia on the 454 FLX system, and MOTU5 on an Illumina HiSeq 2000. Since we were interested in sequence divergence between species, the usage of different platforms will not impair data analyses as might be expected for gene expression studies.

\section{RNA-sequencing and read processing}

For the 454 reads of MOTU3 and $R$. auricularia the script sff_extract (https://bioinf.comav.upv.es/sff_extract/) version 0.3.0, part of the MIRA assembler [61] was used to trim fragments according to the parameters determined by the 454 sequencing pipeline of Roche. MIRA, version 3.4.0, was used for the assembly of the 454 data sets, as simulation results with in silico generated 454 reads show it is one of the best assemblers for the de novo assembly of transcriptomes [62]. MIRA was run using the -accurate quick flag, additionally using the -AS:sep flag to force skimming and recalculating of the Smith-Waterman alignments after each main pass of the algorithm. This was used in combination with the quick settings for the de novo assembly of 454 transcriptomes, -denovo, 454, est. For the manually trimmed instances, where no trace data was available, the option -notrace was used as well.

Adaptor removal and de novo assembly for the Illumina generated reads of $R$. balthica and MOTU5 were conducted using the software CLC Genomics Workbench v3.7 (CLC Bio). The CLC Genomics Workbench uses a de Bruijn graph-based method that is suitable for the efficient assembly of short read data sets with large numbers of reads. For the assembly the bubble size was set to 50 and the kmer size was chosen automatically resulting in a size of 22 for $R$. balthica and 24 for MOTU5.

\section{Mt-genome assembly, verification and annotation}

To reconstruct the mitochondrial genomes for MOTUs 3,5 and $R$. auricularia, each of the RNAseq read sets were searched against the $R$. balthica mt-genome [31] using BlastN. Reads with mt-genome hits were assembled in GENEIOUS v4.8.5 (Biomatters). To close gaps, as well to confirm the accuracy of the assembly, primers were designed using the PRIMER3 web interface (http:// primer3.sourceforge.net/webif.php) (Additional file 7). Due to possible sequencing and assembly errors, we resequenced the mt-genomes of all four species, including $R$. balthica, with the Sanger method. The annotated $R$. balthica mt-genome [31] and pulmonate gene- and rRNA-sequences published in White et al. [63] were used as reference for gene prediction. tRNAs were predicted using the softwares tRNA-scanner [64] and ARWEN (Laslett\&Canbäck 2008). Where necessary, missing tRNAs were annotated manually by alignment of pulmonate tRNA-sequences [63].

\section{Construction of ortholog clusters}

The ortholog search was conducted with HaMStR v9 [34; http://sourceforge.net/projects/hamstr]. HaMStR comes with a set of 1253 so called core-orthologs, i.e., a set of pre-computed clusters of ortholog genes of species with a complete genome available and known phylogeny (species included: Lottia gigantean, Helobdella robusta, Capitella capitata, Schistosoma masoni, Apis mellifera, Daphnia pulex and Caenorhabditis elegans). While the number of possible ortholog-clusters is limited using this program, the main advantage is the small number of false positives [33]. Open reading frames were predicted using genewise [65], a Hidden Markov Model based program which accounts for frameshifts wrongly introduced by sequencing errors using standard parameters with flags set to -trans -cdna-pep-sum. The predicted protein sequences inside each ortholog cluster were aligned using MUSCLE v3.8.31 [66] with standard parameters. These protein alignments were then used as a reference for creating codonwise alignments of the protein encoding nucleotide sequences using a custom Python script, utilizing BioPython [67]. The UTRs of each ortholog cluster were directly aligned using MUSCLE. For all subsequent analyses only sequence alignments with a mean nucleotide sequence divergence of $<10 \%$ between all four species were analyzed to reduce false positives.

\section{Phylogenetic analysis}

Evolutionary relationships among the four Radix species were reconstructed based on the complete mitochondrial 
genomes and 23 nuclear loci. The latter were chosen based on annotated gene clusters, which added up to a length of $14.836 \mathrm{bp}$, comparable to the mt-genomes (13.745 bp - 13.963 bp). To include Biomphalaria glabrata as outgroup, the 23 nuclear loci were searched against the $B$. glabrata gene set provided by VectorBase (https://www.vectorbase.org/) using BLASTx. The B. glabrata mt-genome was obtained from Genbank (accession: AAQ75773.1). The sequences were aligned to the ortholog Radix clusters using BioEdit [68]. MEGA5.4 [69] was used to identify the best fitting model of nucleotide sequence evolution for the mt-genomes and each of the 23 nuclear loci (Additional file 8). We built a Bayesian tree based on the mt-genomes only and one based the mt-genomes plus the nuclear loci using Beast v1.8.0 [70]. This program uses multiple loci to infer a species tree that takes into account stochastic differences in the coalescent histories of the sampled gene genealogies. The analysis was performed with a Yule prior, and run for 10.000.000 generations, sampling every 1000 generations for a total of 10.000 trees. To assess convergence, the effective sample size values and consistency of parameter estimates were monitored using Tracer v1.5 (http://tree.bio.ed.ac.uk/ software/tracer/). From the 10.000 sampled genealogies, the maximum-clade credibility tree was obtained using TreeAnnotator in BEAST, discarding the first 1.000 trees as burn-in.

\section{Selection and functional divergence analyses}

Tests for positive selection were performed using three different methods and tools: a) PAML v4.7 [71], b) a custom version of the McDonald-Kreitman Test implemented in Python using features of the BioPython package (http://www.biopython.org) and c) the program TreeSAAP v3.2 [72]. PAML was used to detect positive selection amongst all four species, while the McDonaldKreitman test was performed on an additional 236 ortholog clusters containing sequences from $R$. balthica and MOTU3 only. In addition, we used TreeSAAP to determine the magnitude of changes in the physiochemical properties of the amino acid resulting from nonsynonymous substitutions among the four species.

To detect signatures of positive selection the program codeml of the PAML package was used to perform a branch model test. codeml estimates the nonsynonymous/synonymous substitution ratio $(\omega=\mathrm{dN} / \mathrm{dS})$, where $\omega=1$ indicates neutral evolution, $\omega<1$ purifying selection, and $\omega>1$ indicates positive selection. Calculation of global $\omega$ values using the one ratio model was followed by calculation of branch-specific $\omega$ using the free ratio model. Each model assigns a log-likelihood to each examined alignment and tree topology. To test for statistical significance log-likelihood ratios were calculated and FDR corrected for multiple testing [73]. The tree topology as inferred by the phylogenetic analyses was used as input for codeml (Additional file 2). This method is quite robust for between species comparisons as it is based on the rate of nonsynonymous to synonymous substitutions [74-76]. Excess nonsynonymous substitutions, accumulate only slowly by sequential selective sweeps [74]. Thus between population variation within species will only have minor effect on the identification of differentially selected genes among species.

Instead of comparing the rates of synonymous to nonsynonymous substitutions, the McDonald-Kreitman Test (MK test) looks into the absolute number of fixed, nonvariable, substitutions $D$ and variable polymorphisms $P$ and into whether those substitutions and polymorphisms are synonymous ( $D s$ and $P s)$ or non-synonymous ( $D n$ and $P n)$. The principle idea underlying this test is the following: Given neutral evolution, the ratio of nonsynonymous to synonymous polymorphisms $P n / P s$ is expected to be the same as the ratio of non-synonymous to synonymous substitutions $D n / D s$ [77]. To obtain substitution and polymorphism information, a custom python script was written to read a pairwise alignment in FASTA format and iterate over each codon present in the alignment. SNPs were called when the read depth at the position of interest was at least 10 and the minor allele frequency was 0.2 without a cutoff for the required base qualities of the reads. Codons with gaps or missing data were ignored. If a given codon differed between both species, it was classified as either non-synonymous $(n)$ or synonymous $(s)$ and either as a polymorphic site $(P)$ or a substitution $(D)$. From these raw counts, the $D n / D s$ and the $P n / P s$ ratios were calculated. Positive selection was assumed if $D n / D s>P n / P s$.

TreeSAAP tests the effect of amino acid changes for up to 31 different physiochemical properties, like bulkiness or polarity. The observed changes and their impact are compared to a distribution expected at random under neutral conditions, with significance determined through a goodness-of-fit test under a $X^{2}$ distribution [72]. TreeSAAP was run with the ortholog clusters concatenated in chunks of 100 clusters per run. In each run, all 31 properties were tested with a window size of 20 codons and the same tree as used for PAML. Again, only clusters with a mean sequence divergence $<10 \%$ were used. To avoid false positives due to sequencing or assembly errors, substitutions were classified as significant when at least two codons were predicted to have an impact class of $\geq 6$ for the physiochemical property changes with a p-value of $<0,001$.

\section{Annotation and enrichment analyses}

All core ortholog-clusters provided by HaMStR are provided with unique gene identifiers based on the $C$. elegans WB190 genome. The according annotation file was 
downloaded from the Gene Ontology website (http:// www.geneontology.org/). We used a custom Python script implemented in the goatools package (https:// github.com/tanghaibao/goatools) to extract all GO terms and the corresponding descriptions from the subontology "biological process" and to map this information to the ortholog clusters. The 1252 HaMStR core ortholog sequences were used as "neutral" reference data set for the enrichment analyses, and various cluster sets as test-set (with signature of selection: all 134 clusters, 112 clusters based on PAML and TreeSAAP including all four species, and $23 M K$-Test clusters based on $R$. balthica and MOTU3 only; without signature of selection: $456 R$. balthica and MOTU3 only; 235 clusters containing sequences of all four species). A one-tailed Fisher's Exact Test was performed in BLAST2GO [78], with a p-value cut-off of 0,05 , after FDR correction for multiple testing [73]. If many of our selected genes are indeed involved in specific functions, we would expect to find these overrepresented in the set of selected genes, but not so in the set of genes without signatures of selection, independent of possible biases. To exclude the possibility of a methodological bias, we not only tested for enriched functional categories in clusters with signatures of selection but also without selective signatures. A possible bias could have resulted from the reference data set (HaMStR core orthologs are evolutionary conserved genes) or the nature of transcriptome obtained contigs, which "only" represent a subset of genes expressed at the time of RNA isolation.

\section{Inference on climatic niche}

To assess climatic niche divergence between the four Radix species, we extracted 35 climatic parameters (e.g., precipitation, various temperature and Bioclim parameters) for each of the 225 sampling points for the period from 1960 - 2000 from publicly available WorldClim data, incorporated in DIVA-GIS [79]. We used a principle component analysis (PCA) based on a correlation matrix to contrast the most important climatic factors between the species niches in Past 3.01 [80].

\section{Data accessibility}

All raw sequence data are deposited as BioProject [GenBank:PRJNA265216]. Ortholog clusters can be retrieved from Dryad [doi:10.5061/dryad.1d49q]. All other data are deposited as supporting information.

\section{Additional files}

Additional file 1: Results of the a) sequencing effort and b) assembly statistics. (PDF $257 \mathrm{~kb}$ )

Additional file 2: Phylogenetic inference on species relationships. (PDF $255 \mathrm{~kb}$ )
Additional file 3: Results and figure for the climate niche divergence PCA. (PDF $14 \mathrm{~kb}$ )

Additional file 4: Selected genes with annotations. (XLSX $35 \mathrm{~kb}$ ) Additional file 5: Results enrichment analyses. (XLSX $11 \mathrm{~kb}$ ) Additional file 6: Snail collection sites. (PDF $330 \mathrm{~kb}$ ) Additional file 7: Radix mt-genome primers. (XLS $36 \mathrm{~kb}$ ) Additional file 8: Annotations and evolutionary models of the clusters used for phylogenetic inference. (XLSX $10 \mathrm{~kb}$ )

\section{Competing interests}

The authors declare that they have no competing interests.

\section{Authors' contributions}

$\mathrm{BF}$ and MP conceived the study and sequenced the transcriptomes. BF and BG assembled the contigs. BG and IE conducted the ortholog search, BG performed the selection analyses. EF and BF sequenced and assembled the mt-genomes. BF and MP have written the manuscript. All authors read and approved the final version of the manuscript.

\section{Acknowledgements}

This work was supported by the research funding programme "LOEWE Landes-Offensive zur Entwicklung Wissenschaftlich-ökonomischer Exzellenz" of Hesse's Ministry of Higher Education, Research, and the Arts. IE acknowledges financial support by the Biodiversity and Climate Research Centre Frankfurt (BIK-F).

\section{Author details}

${ }^{1}$ Molecular Ecology Group, Senckenberg Biodiversity and Climate Research Centre (BiK-F), Georg-Voigt-Str. 14-16, Frankfurt am Main 60325, Germany. 2Evolutionary Biology, Johannes Gutenberg University Mainz, Müllerweg 6, Mainz 55099, Germany. ${ }^{3}$ Applied Bioinformatics Group, Institute of Cell Biology and Neuroscience, Goethe University Frankfurt, Maxvon-Laue Str. 13, Frankfurt am Main 60438, Germany.

Received: 27 April 2015 Accepted: 24 July 2015

Published online: 19 August 2015

\section{References}

1. Butlin R, Debelle A, Kerth C, Snook RR, Beukeboom LW, Castillo Cajas RF, et al. What do we need to know about speciation? Trends Ecol Evol. 2011;27:1-39.

2. Schluter D. Ecology and the origin of species. Trends Ecol Evol. 2001;16:372-80.

3. Rundle HD, Nosil P. Ecological speciation. Ecol Lett. 2005:8:336-52.

4. Keller I, Seehausen O. Thermal adaptation and ecological speciation. Mol Ecol. 2012;21:782-99.

5. Schluter D, Conte GL. Genetics and ecological speciation. Proc Natl Acad Sci U S A. 2009; 106 Suppl (C):9955-62.

6. Stearns SC. The evolution of life histories. Oxford: Ocford University Press; 1992.

7. Roff DA. The evolution of life histories. Theory and analysis. New York: Chapman and Hall; 1992

8. Bale JS. Insects and low temperatures: from molecular biology to distributions and abundance. Philos Trans R Soc Lond B Biol Sci. 2002;357:849-62.

9. Hodkinson ID. Terrestrial insects along elevation gradients: species and community responses to altitude. Biol Rev Camb Philos Soc. 2005;80:489-513.

10. Kolss M, Vijendravarma RK, Schwaller G, Kawecki TJ. Life-history consequences of adaptation to larval nutritional stress in Drosophila. Evolution (N Y) 2009;63:2389-401.

11. Minards NA, Trewick SA, Godfrey AJR, Morgan-Richards M. Convergent local adaptation in size and growth rate but not metabolic rate in a pair of parapatric Orthoptera species. Biol J Linn Soc. 2014;113:123-35.

12. Telfer MG, Hassall M. Ecotypic differentiation in the grasshopper Chorthippus brunneus: life history varies in relation to climate. Oecologia. 1999;121:245-54

13. Schindler D. Widespread effects of climatic warming on freshwater ecosystems in North America. Hydrol Process. 1997;11:1043-67. 
14. Vannote R, Minshall G, Cummins K, Sedell J, Cushing C. The river continuum concept. Can J Fish Aquat Sci. 1980;37:130-7.

15. Caissie D. The thermal regime of rivers: a review. Freshw Biol. 2006:51:1389-406.

16. Ungerer MC, Johnson LC, Herman MA. Ecological genomics: understanding gene and genome function in the natural environment. Heredity (Edinb). 2008;100:178-83.

17. Angilletta MJ. Thermal adaptation - a theoretical and empirical synthesis. Oxford: Oxford University Press; 2009.

18. Li YF, Costello JC, Holloway AK, Hahn MW. "Reverse ecology" and the power of population genomics. Evolution. 2008;62:2984-94.

19. Økland J. Lakes and snails: environment and gastropoda in 1500 Norwegian lakes, ponds and rivers. Oegstegeest, The Netherlands: U.B.S./Dr. W. Backhuys; 1990.

20. Glöer P, Meier-Brook C. Süßwassermollusken. Hamburg: Deutscher Jugendband für Naturbeobachtung; 1998.

21. Schniebs K, Glöer P, Vinarski MV, Hundsdoerfer AK. Intraspecific morphological and genetic variability in Radix balthica (Linnaeus 1758) (Gastropoda: Basommatophora: Lymnaeidae) with morphological comparison to other European Radix species. J Conchol. 2011;40:657-78.

22. Ahlgren J, Yang X, Hansson L, Bronmark C. Camouflaged or tanned: plasticity in freshwater snail pigmentation. Biol Lett. 2013;9:20130464.

23. Pfenninger $M$, Cordellier M, Streit B. Comparing the efficacy of morphologic and DNA-based taxonomy in the freshwater gastropod genus Radix (Basommatophora, Pulmonata). BMC Evol Biol. 2006:6:100.

24. Glöer P. Die Süßwassergastropoden Nord- Und Mitteleuropas, vol. 73. Hackenheim: ConchBooks; 2002

25. Pfenninger $M$, Salinger $M$, Haun T, Feldmeyer B. Factors and processes shaping the population structure and distribution of genetic variation across the species range of the freshwater snail Radix balthica (Pulmonata, Basommatophora). BMC Evol Biol. 2011;11:135.

26. Marti H, Tanner M, Degremont A, Freyvogel T. Studies on the ecology of Bulinus globosus the intermediate host of Schistosoma haematobium in the Ifakara area, Tanzania. Acta Trop. 1985;42:171-87.

27. Dillon RT. The ecology of freshwater molluscs. Cambridge, UK: Cambridge University Press; 2000.

28. Dennis AB, Hellberg ME. Ecological partitioning among parapatric cryptic species. Mol Ecol. 2010;19:3206-25.

29. Pichaud N, Ballard JWO, Tanguay RM, Blier PU. Mitochondrial haplotype divergences affect specific temperature sensitivity of mitochondrial respiration. J Bioenerg Biomembr. 2013;45:25-35.

30. Feldmeyer B, Wheat CW, Krezdorn N, Rotter B, Pfenninger M. Short read Illumina data for the de novo assembly of a non-model snail species transcriptome (Radix balthica, Basommatophora, Pulmonata), and a comparison of assembler performance. BMC Genomics. 2011:12:317.

31. Feldmeyer B, Hoffmeier K, Pfenninger M. The complete mitochondrial genome of Radix balthica (Pulmonata, Basommatophora), obtained by low coverage shot gun next generation sequencing. Mol Phylogenet Evol. 2010:57:1329-33.

32. Hijmans RJ, Cameron SE, Parra JL, Jones PG, Jarvis A. Very high resolution interpolated climate surfaces for global land areas. Int J Climatol. 2005;25:1965-78.

33. Ebersberger I, Strauss S, von Haeseler A. HaMStR: profile hidden markov model based search for orthologs in ESTs. BMC Evol Biol. 2009;9:157.

34. Das J. The role of mitochondrial respiration in physiological and evolutionary adaptation. BioEssays. 2006;28:890-901.

35. Dowling DK, Friberg U, Lindell J. Evolutionary implications of non-neutral mitochondrial genetic variation. Trends Ecol Evol. 2008;23:546-54.

36. Fontanillas P, Dépraz A, Giorgi MS, Perrin N. Nonshivering thermogenesis capacity associated to mitochondrial DNA haplotypes and gender in the greater white-toothed shrew, Crocidura russula. Mol Ecol. 2005;14:661-70.

37. Patel S, Schell T, Eifert C, Feldmeyer B, Pfenninger M. Characterizing a hybrid zone between a cryptic species pair of freshwater snails. Mol Ecol. 2015;24:643-55.

38. Arnquist $\mathrm{G}$. The evolution of animal genitalia: distinguishing between hypotheses by single species studies. Biol J Linn Soc. 1997:60:365-79.

39. Eberhard WG. Why are genitalia good species characters? Proceedings of the Ninth International Congress of Arachnology, Panamá 1983: Washington, D.C.; 1986

40. Mayr E. Animal species and their evolution. Cambridge: Harvard University Press; 1963.
41. Eberhard WG. Evolution of genitalia: theories, evidence, and new directions. Genetica. 2010;138:5-18

42. Schniebs K, Glöer P, Vinarski MV, Hundsdoerfer AK. Intraspecific morphological and genetic variability in the European freshwater snail Radix labiata (Rossmaessler, 1835) (Gastropoda: Basommatophora: Lymnaeidae). Contrib to Zool. 2013;82:55-68.

43. Rudolf $V$, Rödel M. Phenotypic plasticity and optimal timing of metamorphosis under uncertain time constraints. Evol Ecol. 2007:21:121-42.

44. Smith KK. Time' s arrow: heterochrony and the evolution of development. Int J Dev Biol. 2003;621:613-21.

45. Keyte AL, Smith KK. Heterochrony and developmental timing mechanisms: changing ontogenies in evolution. Semin Cell Dev Biol. 2014;34:99-107.

46. Carroll SB. Endless forms most beautiful: the new science of evo-devo. New York: W.W. Norton \& Co.; 2005

47. Smirthwaite J, Rundle SD, Spicer JI. The use of developmental sequences for assessing evolutionary change in gastropods. Am Malacol Bull. 2009;27:105-11.

48. Tills $O$, Rundle SD, Salinger M, Haun T, Pfenninger M, Spicer Jl. A genetic basis for intraspecific differences in developmental timing? Evol Dev. 2011;13:542-8

49. Fanara JJ, Folguera G, Iriarte PF, Mensch J, Hasson E. Genotype by environment interactions in viability and developmental time in populations of cactophilic Drosophila. J Evol Biol. 2006:19:900-8.

50. Mensch J, Lavagnino N, Carreira VP, Massaldi A, Hasson E, Fanara JJ. Identifying candidate genes affecting developmental time in Drosophila melanogaster: pervasive pleiotropy and gene-by-environment interaction. BMC Dev Biol. 2008:8:78

51. Schierenberg E. Embryological variation during nematode development WormBook. 2006;1-13.

52. Tahseen Q. Nematodes in aquatic environments: adaptations and survival, Biodivers J. 2012;3:13-40

53. Angilletta MJ, Oufiero CE, Sears MW. Thermal adaptation of maternal and embryonic phenotypes in a geographically widespread ectotherm. Int Congr Ser. 2004;1275:258-66.

54. Van Den Biggelaar J, Haszprunar G. Cleavage patterns and mesentoblast formation in the Gastropoda: an evolutionary perspective. Evolution (N Y). 1996;50:1520-40.

55. Gervasi SS, Foufopoulos J. Costs of plasticity: responses to desiccation decrease post-metamorphic immune function in a pond-breeding amphibian. Funct Ecol. 2008;22:100-8.

56. Varela-Lasheras I, Van Dooren TJ. Desiccation plasticity in the embryonic life histories of non-annual rivulid species. Evodevo. 2014:5:16.

57. Cridland CC. Resistance of Bulinus (Physopsis) globosus, Bulinus (Ph.) africanus, Biomphalaria pfeifferi and Lymnaea natalensis to experimental desiccation. Bull World Health Organ. 1967;36:507-13.

58. Facon B, MacHline E, Pointier JP, David P. Variation in desiccation tolerance in freshwater snails and its consequences for invasion ability. Biol Invasions. 2004;6:283-93.

59. Chapuis E, Ferdy J-B. Life history traits variation in heterogeneous environment: the case of a freshwater snail resistance to pond drying. Eco Evol. 2012;2:218-26.

60. Baur B, Raboud C. Life history of the land snail Arianta arbustorum along an altitudinal gradient. J Anim Ecol. 2008:57:71-87.

61. Chevreux B, Pfisterer T, Drescher B, Driesel AJ, Mller WEG, Wetter T. Using the miraEST assembler for reliable and automated mRNA transcript assembly and SNP detection in sequenced ESTs. Genome Res. 2004;14:1147-59.

62. Mundry M, Bornberg-Bauer E, Sammeth M, Feulner PGD. Evaluating characteristics of de novo assembly software on 454 transcriptome data: a simulation approach. PLoS One. 2012;7, e31410.

63. White TR, Conrad MM, Tseng R, Balayan S, Golding R, de Frias Martins AM, et al. Ten new complete mitochondrial genomes of pulmonates (Mollusca: Gastropoda) and their impact on phylogenetic relationships. BMC Evol Biol. 2011;11:295

64. Lowe TM, Eddy SR. tRNAscan-SE: a program for improved detection of transfer RNA genes in genomic sequence. Nucleic Acids Res. 1997;25:955-64.

65. Birney E, Clamp M, Durbin R. GeneWise and Genomewise. Genome Res. 2004;14:988-95.

66. Edgar RC. MUSCLE: multiple sequence alignmetn with high accuracy and high throughput. Nucleic Acids Res. 2004;32:1792-7. 
67. Cock PJA, Antao T, Chang JT, Chapman BA, Cox CJ, Dalke A, et al. Biopython: freely available Python tools for computational molecular biology and bioinformatics. Bioinformatics. 2009;25:1422-3.

68. Hall TA. BioEdit: a user-friendly biological sequence alignment editor and analysis program for Windows 95/98/NT. Nucleic Acids Symp Ser. 1999:41:95-8.

69. Kumar S, Nei M, Dudley J, Tamura K. MEGA: a biologist-centric software for evolutionary analysis of DNA and protein sequences. Brief Bioinform. 2008:9:299-306.

70. Drummond AJ, Suchard MA, Xie D, Rambaut A. Bayesian phylogenetics with BEAUti and the BEAST 1.7. Mol Biol Evol. 2012;29:1969-73.

71. Yang Z. PAML 4: phylogenetic analysis by maximum likelihood. Mol Biol Evol. 2007:24:1586-91.

72. Woolley S, Johnson J, Smith MJ, Crandall KA, McClellan DA. TreeSAAP: Selection on Amino Acid Properties using phylogenetic trees. Bioinformatics. 2003;19:671-2

73. Benjamni $Y$, Hochberg $Y$, Benjamini $Y$. Controlling the false discovery rate: a practical and powerful approach to multiple testing. J R Stat Soc B. 1995;57:289-300.

74. Hohenlohe PA, Phillips PC, Cresko WA. Using population genomics to detect selection in natural populations: key concepts and methodological considerations. Int J Plant Sci. 2010;171:1059-71.

75. Yang Z, Bielawski JP. Statistical methods for detecting molecular adaptation. TREE. 2000;15:496-503.

76. Yang Z, Nielsen R, Goldman N, Pedersen A-MK. Codon-substitution models for heterogeneous selection pressure at amino acid sites. Genetics. 2000;155:431-49.

77. Kreitman M. Methods to detect selection in populations with applications to the human. Annu Rev Genomics Hum Genet. 2000;1:539-59.

78. Conesa A, Gotz S, Garcia-Gomez JM, Terol J, Talon M, Robles M. Blast2GO: a universal tool for annotation, visualization and analysis in functional genomics research. Bioinformatics. 2005;21:3674-6.

79. Hijmans RJ, Guarino L, Cruz M, Rojas E. Computer tools for spatial analysis of plant genetic resources data: 1. DIVA-GIS. Plant Genet Resour Newsl. 2001;127:15-9.

80. Hammer $\varnothing$, Harper DAT, Ryan PD. Paleontological statistics software package for education and data analysis. Palaeontol Electron. 2001;4:9-18.

\section{Submit your next manuscript to BioMed Central and take full advantage of:}

- Convenient online submission

- Thorough peer review

- No space constraints or color figure charges

- Immediate publication on acceptance

- Inclusion in PubMed, CAS, Scopus and Google Scholar

- Research which is freely available for redistribution 